\title{
CASPASE-DEPENDENT ACTIVATION OF CASCADE CYTOKINES IN YOUNG PATIENTS WITH AUTOIMMUNE THYROIDITIS IN COMBINATION WITH GASTROESOPHAGEAL REFLUX DISEASE
}

\author{
Tamara Pasiieshvili \\ Department of Internal Medicine No. 3 and Endocrinology ${ }^{1}$ \\ pasotoma2017@gmail.com \\ Lyudmila Pasiieshvili \\ Department of General Practice-Family Medicine and Internal Diseases ${ }^{1}$ \\ pasiieshvili@gmail.com \\ Natalia Zhelezniakova \\ Department of Internal Medicine No. $1^{1}$ \\ Nataliya2509@gmail.com \\ Olga Kovalyova \\ Department of General Practice-Family Medicine and Internal Diseases ${ }^{1}$ \\ prokov@gmail.com \\ ${ }^{1}$ Kharkiv National Medical University \\ 4 Nauky ave., Kharkiv, Ukraine, 61022
}

\begin{abstract}
The aim of the study: to assess the role of caspase-1 and IL-18 in the implementation of the inflammatory response in young patients with autoimmune thyroiditis (AIT) associated with gastroesophageal reflux disease (GERD).

Materials and methods. The study was conducted in two groups of patients homogeneous by gender and age. The first group included 42 patients with an isolated AIT and the second group - 120 patients with a comorbid course of AIT and GERD. The contingent of the surveyed was students. Median age was from 18 to 25 years: $23.1 \pm 1.2$ years in group with isolated AIT and $21.9 \pm 2.7$ years in group with combined pathology. The activity of caspase-1 was determined using «Elabscience» kits, USA (China) by immunoassay method. The content of IL-18 was investigated using commercial kits «Bender MedSystems GmbH» (Austria) according to the proposed methods by immunoassay method. Statistical data processing was carried out using the local universal software package Statistica Basic Academic 13 for Windows En Local. Methods of non-parametric statistics were used: Kruskal-Wallis test, median test, Mann-Whitney test.

Results. It has been found that caspase- 1 is activated in patients both in the isolated course of AIT and in its combination with GERD. At the same time, a significant increasing in the synthesis of IL-18 was established, which has pro-inflammatory and autoimmune effects. Re-examination after 2 months of caspase-1 and IL-18 in examined persons determined decreasing their levels, but control results were not achieved.

Conclusion. In young patients, the combination of GERD and AIT, as well as an isolated AIT is accompanied by an increasing the levels of caspase-1 and IL-18 in blood serum related to inflammatory process in the thyroid gland and esophageal mucosa with autoimmune component. These biomarkers may reflect the severity of clinical course of diseases and serve as prognostic indicator of outcome in case of comorbidity.
\end{abstract}

Keywords: autoimmune thyroiditis, gastroesophageal reflux disease, pathogenesis, caspase-1, interleukin-18.

DOI: 10.21303/2504-5679.2021.001603

\section{Introduction}

The development of an inflammatory reaction in any disease of internal organs obeys to the basic law of inflammation - the participation of the immune system in its implementation. The sequence of the inclusion of its various components, the volume of the cellular and humoral response depends on many factors: the action and the virulence of the pathogen, the state of 
the immune system both in general and its individual links, concomitant diseases and a number of others [1].

An inflammatory reaction is a genetically determined function of the body, aimed at maintaining homeostasis, that is, neutralizing the pathogen without significant consequences for the body $[1,2]$.

The cytokine cascade is triggered by the activation of an caspase-1, which is precursor to all inflammatory states. An increasing of the of caspase-1 level starts the inflammatory reaction, as well as activates and expands it due to the synthesis of cytokines - interleukins (IL) - IL-1 $\beta$, IL-6 and IL-18 [3-5].

The development of autoimmune thyroiditis (AIT) was considered from the position of impaired immunogenesis. This theory is supplemented by the participation of a complex of cytokine and chemokine-mediated reactions. Lymphocytic infiltration of the thyroid gland in patients with AIT occurs against the background of a «cytokine explosion», since the lymphocytes themselves are the main producers of these information biomolecules [6, 7].

The combination of AIT with various diseases of internal organs creates an unfavorable autoimmune background, the presence of which contributes to the prolongation of the concomitant disease, lengthening the period of exacerbation and the formation of defective remission. Among such nosological forms, gastroesophageal reflux disease (GERD) is considered as one of the socially significant diseases that has an adverse effect on the quality of patients life $[8,9]$.

The aim of the research was to assess the role of caspase-1 and IL-18 in the implementation of the inflammatory response in young patients with AIT associated with GERD.

\section{Materials and methods}

Study was conducted in 2017-2019 years on the basis of the Municipal Nonprofit Enterprise of Kharkiv Town Council «City Student Hospital» (Kharkiv) during, Ukraine. All study participants were students and were divided into two groups. The first group included 42 patients ( 35 women and 7 men), average age $23.1 \pm 1.2$ years with AIT. The second group contained from 120 patients with a combination of AIT and GERD (93 women and 27 men), average age $21.9 \pm 2.7$ years. The control group consisted of 20 (15 women and 5 men) practically healthy people, average age $22.4 \pm 2.3$ years.

Recommendation of the Montreal Consensus (2006) and ICD-10 were used for verification of the GERD diagnosis. Esophagogastroduodenoscopy (EFGDS) was made for determination of morphological forms of GERD; Los Angeles classification was used. The autoimmune nature of thyroiditis was confirmed by the increased levels of antibodies to thyroid peroxidase and thyroglobulin. The hormonal status of the thyroid gland was assessed by the levels of thyroxine, triiodothyronine and thyroid-stimulating hormone.

The study conducted in accordance with treatment standards of the requirements for the ethical component of clinical trials (GCP, 1997). It was approved by ethics committee of Kharkiv National Medical University (protocol No. 11 of 05.12.2018). All patients signed informed written consent for participating in the clinical study.

The activity of caspase-1 was determined using «Elabscience» kits, USA (China) by immunoassay method. The content of IL-18 was investigated using commercial kits «Bender MedSystems GmbH» (Austria) according to the proposed methods by immunoassay method.

Statistical processing was carried out by the method of variation statistics using the standard software Statistica Basic Academic 13 for Windows En Local. Methods of non-parametric statistics were used: Kruskal-Wallis test, median test, Mann-Whitney test. The difference was considered as significant at $p<0.05$.

\section{Results}

When analyzing the obtained data was found that the activity caspase- 1 in patients with AIT is significance higher compare to the control group. In patients with a combination of GERD and AIT, the level of caspase-1 significantly exceeded both the norm and the group with isolated AIT (Table 1). 
It can be assumed that the higher level of caspase-1 in patients with comorbid pathology are not a consequence of a simple addition of the activity of two diseases, but are due to an increase in the inflammatory component in GERD by an unfavorable autoimmune background [10, 11].

Table 1

The levels of caspase-1 and IL-18 in the serum blood of the examined persons

\begin{tabular}{lcc}
\hline \multicolumn{1}{c}{ Groups/parametrs } & Caspase-1, pg/ml & IL-18, pg/ml \\
\hline AIT $(n=42)$ & $688.5712(534.1920 ; 964.3711)$ & $1029.20(779.30 ; 1252.40)$ \\
AIT with GERD $(n=120)$ & $1394.2840(1155.1980 ; 1517.1320)$ & $1763.40(1451.70 ; 2879.20)$ \\
Control group $(n=20)$ & $510.7821(311.8725 ; 618.3146)$ & $229.40(198.31 ; 269.37)$ \\
& & \\
Significance of difference & $U_{1-3}=203 ; p_{1-3}<0.01$ & $U_{1-3}=552 ; p_{1-3}<0.01$ \\
& $U_{1-2}=645 ; p_{1-2}<0.01$ & $U_{1-2}=313 ; p_{1-2}<0.01$ \\
& $U_{2-3}=102.5 ; p_{2-3}<0.01$ & $U_{2-3}=223 ; p_{2-3}<0.01$
\end{tabular}

Note: ${ }^{1}-p<0.05$ - the difference is statistically significant; $U_{1-3}, p_{1-3}-$ the difference between the group with isolated AIT and control group; $U_{1-2}, p_{1-2}-$ the difference between the group GERD+AIT and the isolated group AIT; $U_{2-3}, p_{2-3}-$ the difference between the group GERD+AIT and the control group

The launch of the cytokine cascade through the activation of caspase-1 provides that the content of IL-18 will increase. So it was found that the activity of IL-18 significantly exceeded the control indicators in both examined groups. It has been established that IL-18 has not only a pro-inflammatory effect, but can also act as a marker of autoimmune processes, which is confirmed by its active synthesis in patients with AIT $[12,13]$.

Thus, both in individuals with isolated AIT and in its combination with GERD, an increasing the levels of caspase- 1 and IL-18 was noted as a result of an active inflammatory process in the esophageal mucosa and thyroid tissue.

The follow-up period for two months in 34 patients with a combined course of AIT and GERD and 19 - with isolated AIT showed that the level of caspase-1 in the group with combined pathology exceeded the control values; however, compared with initial level, a decrease in caspase-1 level was noted (Fig. 1). The activity of caspase-1 did not change in the group with isolated AIT compared with the initial figures.

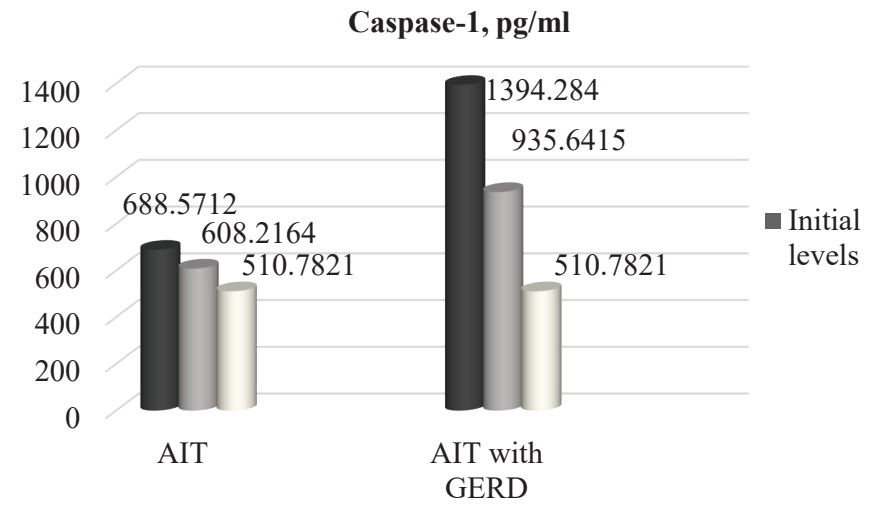

Fig. 1. The levels of caspase-1 in the serum blood of the examined patients, $\mathrm{pg} / \mathrm{ml}$

Evaluation of IL-18 indicators in dynamics showed that the increased level remained in the group with combined pathology, but this parameter was 2 times less than the baseline characteristic. There is no significant difference of IL-18 level in group with isolated AIT (Fig. 2).

Changes in caspase-1 and IL-18 levels in dynamics of baseline characteristic and follow-up time two months are presented in Table 2. 


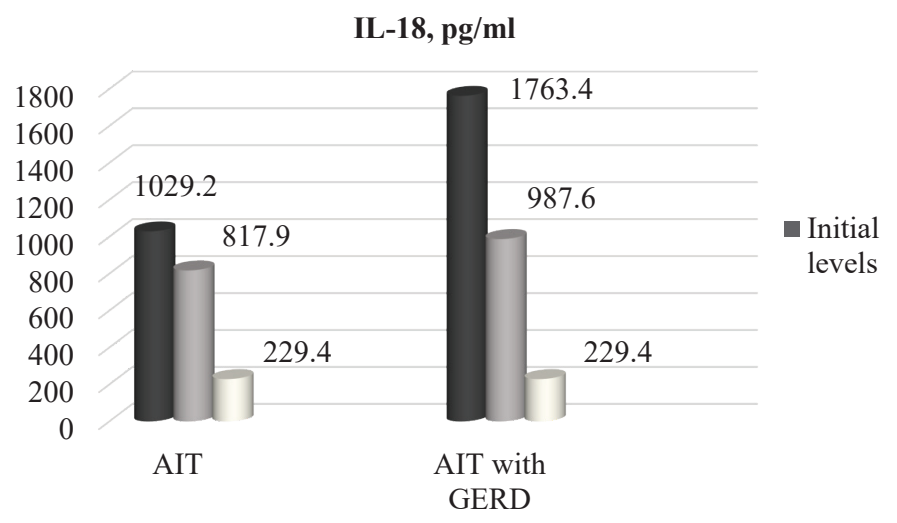

Fig. 2. The levels of IL-18 in the serum of the examined patients, $\mathrm{pg} / \mathrm{ml}$

Table 2

Dynamics of caspase-1 and IL-18 in patients

\begin{tabular}{|c|c|c|c|}
\hline \multirow{2}{*}{ Groups } & & \multicolumn{2}{|c|}{ Indicators } \\
\hline & & Caspase-1, pg/ml & IL-18, pg/ml \\
\hline \multirow[t]{2}{*}{ AIT with GERD $(n=34)$} & Initial levels & $1394.2840(1155.1980 ; 1517.1320)$ & $1763.4(1451.7 ; 2879.2)$ \\
\hline & In 2 month & $935.6415(812.1261 ; 1171.2125)$ & $987.6(876.5 ; 1254.3)$ \\
\hline \multirow[t]{2}{*}{$\operatorname{AIT}(n=19)$} & Initial levels & $688.5712534 .1924 ; 964.3711)$ & $1029.2(779.3 ; 1252.4)$ \\
\hline & In 2 month & $608.2164(487.5431 ; 804.4362)$ & $817.9(783,9 ; 952.4)$ \\
\hline
\end{tabular}

The persistence of increased results of these indicators after 2 months in patients with AIT, in our opinion, can be explained by the continuous, latent autoimmune mechanism of thyroiditis. In patients with the combined pathology of AIT and GERD, these changes, apparently, on the one hand, are also associated with the absence of immunological remission, on the other hand, they can be explained by the persistence of moderate foci of inflammation in the mucous membrane and the development of endothelial dysfunction.

\section{Discussion}

The combined course of GERD and AIT in young patients is accompanied by the formation of systemic inflammation, which is confirmed by increased synthesis of caspase-1 and IL-18. Assessment of indicators of caspase-1 and IL-18 in dynamics showed a decrease in the levels of these parameters compared to the initial levels, but the control figures were not achieved. This circumstance, in our opinion, can be explained by the latent autoimmune mechanism of thyroiditis and the persistence of moderate foci of inflammation in the mucous membrane.

Caspase-1 is considered as a pro-inflammatory mediator in GERD. Under conditions of alcohol-induced esophagitis in cell culture and in vivo, was found caspase-1 activation and DNA fragmentation [14]. Caspase-1 converts inactive pro-IL-1 $\beta$ and pro-IL-18 into active pro-inflammatory cytokines, enhances the inflammatory response, induces pyroptosis an inflammatory cell death after its activation various inflammasomes, thus pyroptosis was involved in the occurrence of esophagitis $[15,16]$. The use of an inhibitor of caspase-1 could suppress the activity of IL-1 and IL-18 in vivo and in vitro reducing the inflammatory response so may considered as future therapeutic target [14]. Similar results in determining the role of caspase-1 were obtained in an animal model of esophagitis induced by the introduction of bile acid salts [17]. Guo Q. with coauthors (2018) suggested that the release of active IL-18 from thyroid cells increases the immune response in thyroid tissues in patients with AIT [18]. IL-18 promotes the activation of the inflammatory cascade, which leads to the destruction of the gland in AIT [19]. 
Study limitations. The study was not conducted in case of identified concomitant pathology of other organs and in case of patients refuse to participate in the study.

Prospects for further research. In subsequent works, further identification of other pathogenetic links of the GERD and AIT is assumed.

\section{Conclusions}

1. The GERD associated with AIT in young patients is accompanied by an increase in the synthesis of caspase-1 and IL-18 in serum blood related to inflammatory process in the thyroid gland and esophageal mucosa with autoimmune component.

2. Isolated AIT in young people leads to an increase in caspase-1 and IL-18 levels, which confirms the latent nature of autoimmune inflammation in the thyroid gland.

3. Assessment of indicators of caspase-1 and IL-18 in dynamics in patients with GERD and AIT, as well as in group with isolated AIT, showed a decrease in the levels of these parameters compared to the initial levels, but the control figures were not achieved.

4. Caspase-1 and IL-18 can be used as biomarkers of the severity of the clinical course of pathologies and serve as a prognostic indicator of outcome in the case of concomitant pathology.

\section{Conflict of interests}

The authors declare that they have no conflicts of interest.

\section{References}

[1] Chen, L., Deng, H., Cui, H., Fang, J., Zuo, Z., Deng, J. et. al. (2017). Inflammatory responses and inflammation-associated diseases in organs. Oncotarget, 9 (6), 7204-7218. doi: http://doi.org/10.18632/oncotarget.23208

[2] Zhao, Y., Forst, C. V., Sayegh, C. E., Wang, I. M., Yang, X., Zhang, B. (2016). Molecular and genetic inflammation networks in major human diseases. Molecular bioSystems, 12 (8), 2318-2341. doi: http://doi.org/10.1039/c6mb00240d

[3] Rasmussen, G., Idosa, B. A., Bäckman, A., Monecke, S., Strålin, K., Särndahl, E., Söderquist, B. (2019). Caspase-1 inflammasome activity in patients with Staphylococcus aureus bacteremia. Microbiology and immunology, 63 (12), 487-499. doi: http://doi.org/10.1111/1348-0421.12738

[4] Pfeiler, S., Winkels, H., Kelm, M., Gerdes, N. (2019). IL-1 family cytokines in cardiovascular disease. Cytokine, $122,154215$. doi: http://doi.org/10.1016/j.cyto.2017.11.009

[5] Udjus, C., Cero, F. T., Halvorsen, B., Behmen, D., Carlson, C. R., Bendiksen, B. A. et. al. (2019). Caspase-1 induces smooth muscle cell growth in hypoxia-induced pulmonary hypertension. American journal of physiology. Lung cellular and molecular physiology, 316 (6), L999-L1012. http://doi.org/10.1152/ajplung.00322.2018

[6] Ferrari, S. M., Ruffilli, I., Elia, G., Ragusa, F., Paparo, S. R., Patrizio, A. et. al. (2019). Chemokines in hyperthyroidism. Journal of clinical \& translational endocrinology, 16, 100196. doi: http://doi.org/10.1016/j.jcte.2019.100196

[7] Ragusa, F., Fallahi, P., Elia, G., Gonnella, D., Paparo, S. R., Giusti, C. et. al. (2019). Hashimotos' thyroiditis: Epidemiology, pathogenesis, clinic and therapy. Best Practice \& Research Clinical endocrinology \& metabolism, 33 (6), 101367. doi: http:// doi.org/10.1016/j.beem.2019.101367

[8] Clarrett, D. M., Hachem, C. (2018). Gastroesophageal Reflux Disease (GERD). Missouri medicine, 115 (3), $214-218$.

[9] Fill Malfertheiner, S., Seelbach-Göbel, B., Costa, S. D., Ernst, W., Reuschel, E., Zeman, F. et. al. (2017). Impact of gastroesophageal reflux disease symptoms on the quality of life in pregnant women: a prospective study. European journal of gastroenterology \& hepatology, 29 (8), 892-896. doi: http://doi.org/10.1097/meg.0000000000000905

[10] Lee, D. J., Du, F., Chen, S. W., Nakasaki, M., Rana, I., Shih, V. et. al. (2015). Regulation and Function of the Caspase-1 in an Inflammatory Microenvironment. The Journal of investigative dermatology, 135 (8), 2012-2020. doi: http://doi.org/ 10.1038/jid.2015.119

[11] Bolívar, B. E., Vogel, T. P., Bouchier-Hayes, L. (2019). Inflammatory caspase regulation: maintaining balance between inflammation and cell death in health and disease. The FEBS journal, 286 (14), 2628-2644. doi: http://doi.org/10.1111/febs.14926

[12] Esmailbeig, M., Ghaderi, A. (2017). Interleukin-18: a regulator of cancer and autoimmune diseases. European cytokine network, 28 (4), 127-140. doi: http://doi.org/10.1684/ecn.2018.0401

[13] Yasuda, K., Nakanishi, K., Tsutsui, H. (2019). Interleukin-18 in Health and Disease. International journal of molecular sciences, 20 (3), 649. doi: http://doi.org/10.3390/ijms20030649

[14] Wang, F., Li, G., Ning, J., Chen, L., Xu, H., Kong, X. et. al. (2018). Alcohol accumulation promotes esophagitis via pyroptosis activation. International journal of biological sciences, 14 (10), 1245-1255. doi: http://doi.org/10.7150/ijbs.24347 
[15] Sollberger, G., Strittmatter, G. E., Garstkiewicz, M., Sand, J., Beer, H. D. (2014). Caspase-1: the inflammasome and beyond. Innate immunity, 20 (2), 115-125. doi: http://doi.org/10.1177/1753425913484374

[16] Conos, S. A., Lawlor, K. E., Vaux, D. L., Vince, J. E., Lindqvist, L. M. (2016). Cell death is not essential for caspase-1-mediated interleukin-1 $\beta$ activation and secretion. Cell death and differentiation, 23 (11), 1827-1838. doi: http://doi.org/ $10.1038 /$ cdd. 2016.69

[17] Yin, X. L., Wu, H. M., Zhang, B. H., Zhu, N. W., Chen, T., Ma, X. X. et. al. (2020). Tojapride prevents CaSR-mediated NLRP3 inflammasome activation in oesophageal epithelium irritated by acidic bile salts. Journal of cellular and molecular medicine, 24 (2), 1208-1219. doi: http://doi.org/10.1111/jcmm.14631

[18] Guo, Q., Wu, Y., Hou, Y., Liu, Y., Liu, T., Zhang, H. et. al. (2018). Cytokine Secretion and Pyroptosis of Thyroid Follicular Cells Mediated by Enhanced NLRP3, NLRP1, NLRC4, and AIM2 Inflammasomes Are Associated With Autoimmune Thyroiditis. Frontiers in immunology, 9, 1197. doi: http://doi.org/10.3389/fimmu.2018.01197

[19] Liu, Z., Wang, H., Xiao, W., Wang, C., Liu, G., Hong, T. (2010). Thyrocyte interleukin-18 expression is up-regulated by interferon- $\gamma$ and may contribute to thyroid destruction in Hashimoto's thyroiditis. International journal of experimental pathology, 91 (5), 420-425. doi: http://doi.org/10.1111/j.1365-2613.2010.00715.x 